\title{
Evolution of Globular Cluster Populations in Compact Galaxy Groups
}

\author{
S. Leon, J. Perea, A. Del Olmo \\ Instituto de Astrofísica de Andalucía (CSIC), Granada, Spain \\ E. Athanassoula
}

Observatoire de Marseille, Marseille, France

G. Bergond

MSU Physics and Astronomy Dept.,Lansing, USA

G. Meylan

Space Telescope Science institute, Baltimore, USA

C. Garcia Gomez

Universitat Rovira i Virgili, Tarragona, Spain

\begin{abstract}
The redistribution of globular clusters within compact groups of galaxies is followed through $\mathrm{N}$-body simulations. Particular emphasis is given to the globular clusters released in the Intra-Group Medium (IGM) and to the final configuration of the evolution.
\end{abstract}

\section{Simulations and Results}

We follow the evolution of the GCs in compact groups with 5 Milky Way-like galaxies. The simulations have been done on a PC cluster using the GADGET code. The different runs have the same initial spatial distribution, and only the individual galaxy velocities are changed with increasing virial $(2 \mathrm{~T} / \mathrm{V})$ parameter. The GCs are distributed according to a radial density profile which fits the Galactic population (Djorgovski \& Meylan, 1994, AJ, 108, 1292). Up to 50\% of the initial GC population is lost by the host galaxies after few Gyrs. The GCs loss is sensitive to the initial GC concentration. The final radial distribution of GCs is flatter than the initial one with a drop of the radial slope of 0.2-0.4 for the galaxies that merge, whereas for interacting galaxies the radial distribution can become slightly steeper. The specific frequency SN in most of the runs is decreasing down by $20 \%$ at the most. An important population of GCs is released in the IGM: 200-550 GCs are populating the IGM, with an extension to 200-300 kpc from the center of the group. The distribution of GCs is similar to what is found in the particular case of HCG90, with tidal tails and following the diffuse emission distribution. 